\title{
Lead Site V4
}

National Cancer Institute

\section{Source}

National Cancer Institute. Lead Site V4. NCI Thesaurus. Code C90416.

A unipolar electrocardiog ram lead site; the electrode is placed at the fifth intercostal space on the anterior chest wall (between ribs 5 and 6 ) at the left midclavicular line. In small animals it corresponds to V4-V6 where it is placed at the 6th left intercostal space near the costochondral junction. In large animals it is placed over the 6th rib at the level of a horizontal line drawn through the scapulohumeral articulation on the left side of the thorax. 